\title{
Junction Type Representations of the Temperley-Lieb Algebra and Associated Symmetries
}

\author{
Anastasia DOIKOU ${ }^{\dagger}$ and Nikos KARAISKOS $\dagger$ \\ $\dagger$ Department of Engineering Sciences, University of Patras, GR-26500 Patras, Greece \\ E-mail:adoikou@upatras.gr,nkaraiskos@upatras.gr \\ $¥$ Centre de Physique Théorique, Ecole Polytechnique, CNRS-UMR 7644, \\ 91128 Palaiseau, France
}

Received September 06, 2010, in final form November 29, 2010; Published online December 07, 2010 doi:10.3842/SIGMA.2010.089

\begin{abstract}
Inspired by earlier works on representations of the Temperley-Lieb algebra we introduce a novel family of representations of the algebra. This may be seen as a generalization of the so called asymmetric twin representation. The underlying symmetry algebra is also examined and it is shown that in addition to certain obvious exact quantum symmetries non trivial quantum algebraic realizations that exactly commute with the representation also exist. Non trivial representations of the boundary Temperley-Lieb algebra as well as the related residual symmetries are also discussed. The corresponding novel $R$ and $K$ matrices solutions of the Yang-Baxter and reflection equations are identified, the relevant quantum spin chain is also constructed and its exact symmetries are studied.
\end{abstract}

Key words: quantum integrability; Temperley-Lieb algebras; symmetries associated to integrable models

2010 Mathematics Subject Classification: 81R50; 17B37; 17B80

\section{Introduction}

There has been an increasing activity in recent years on the study of representations of (affine) Hecke algebras $[2,3,4,5,6,7,8,9,10,11,12,13,14,15,16,17,18,19,20,21]$ and their quotients, which can provide solutions [12, 13, 14, 15, 16, 17, 18, 19, 20] of the Yang-Baxter [1] and the reflection equations $[4,23]$. Such investigations are of great physical significance due to the fact that the obtained solutions can be implemented into physical integrable systems, and conformal field theories with open boundary conditions that preserve integrability (see e.g. $[7,8,15,20,22])$, or lead to the construction of novel physical systems as will be transparent in the present investigation (see also [7]). In the present article, we focus on the investigation of novel representations in the context of the Temperley-Lieb (TL) algebra [24, 18], the so called junction representation. In fact, this may be seen as a natural generalization of the asymmetric twin (or cable) representation studied in $[17,7,6]$. More precisely, the representation is introduced and it is shown that it satisfies the defining relations of the algebra, provided that certain constraints are satisfied. In addition, the existence of non trivial quantum algebras that are exact symmetries of the aforementioned representation is investigated. Our analysis is further extended in the case of the boundary Temperley-Lieb (blob) algebra, and non trivial representations for the "boundary" element of the algebra are identified.

It is worth noting that the representation leads naturally to novel physical integrable systems quantum spin chains, with non-trivial integrable boundary conditions. It is thus clear that the whole analysis presents not only mathematical, but physical interest as well, given that such

\footnotetext{
${ }^{\star}$ This paper is a contribution to the Proceedings of the International Workshop "Recent Advances in Quantum Integrable Systems". The full collection is available at http://www.emis.de/journals/SIGMA/RAQIS2010.html
} 
investigations provide new boundary conditions that may alter the physical behavior as well as the symmetries associated to the corresponding system (see e.g. [7, 8, 6]).

The outline of this article is as follows: in the next section the definitions of the TemperleyLieb algebra are reviewed, the junction representation is introduced, and it is shown that it satisfies the Temperley-Lieb algebra defining relations. The exact symmetries associated to the junction representation are then examined, certain obvious and non-trivial representations of quantum algebras are introduced, and turn out to exactly commute with all the elements of the Temperley-Lieb algebra in the junction representation. Explicit proof for the first non trivial case is provided, and expressions of a whole family of representations of the quantum algebra for the generic case are conjectured. In Section 3 the boundary Temperley-Lieb algebras and the related representations are discussed, and the residual symmetries are examined. In the last section we introduce the relevant quantum spin chain, and we also examine the associated symmetries of the open transfer matrix. In the Appendices various technical points are illustrated.

\section{Temperley-Lieb algebra and representations}

Let us briefly review the basic notions associated to the Temperley-Lieb algebra [24, 18]. The Temperley-Lieb $T_{N}(q)$ algebra is defined by the generators $\mathbb{U}_{i}, i=1, \ldots, N$ satisfying

$$
\begin{aligned}
& \mathbb{U}_{i}^{2}=-\left(q+q^{-1}\right) \mathbb{U}_{i}, \quad \mathbb{U}_{i} \mathbb{U}_{i \pm 1} \mathbb{U}_{i}=\mathbb{U}_{i}, \\
& {\left[\mathbb{U}_{i}, \mathbb{U}_{j}\right]=0, \quad|i-j|>1 .}
\end{aligned}
$$

A well known representation of the Temperley-Lieb algebra is the XXZ one. More precisely, consider the matrix

$$
U=\sum_{a \neq b=1}^{2} e_{a b} \otimes e_{b a}-\sum_{a \neq b=1}^{2} q^{-\operatorname{sgn}(a-b)} e_{a a} \otimes e_{b b},
$$

where the matrices $e_{a b}$ are in this case $2 \times 2$ matrices defined as: $\left(e_{a b}\right)_{c d}=\delta_{a c} \delta_{b d}$. Then take the representation of the Temperley-Lieb algebra $\rho: T_{n}(q) \mapsto \operatorname{End}\left(\left(\mathbb{C}^{2}\right)^{\otimes N}\right)$ such that

$$
\rho\left(\mathbb{U}_{i}\right)=\mathbb{I} \otimes \cdots \otimes \mathbb{I} \otimes \underbrace{U}_{i, i+1} \otimes \mathbb{I} \otimes \cdots \otimes \mathbb{I} .
$$

Inspired by the asymmetric twin (or cable) representation [17, 7] of the Temperley-Lieb algebra we introduce a novel representation, which we shall call for obvious reasons the junction representation. This involves essentially $n$ copies of the XXZ representation i.e. $\Theta: T_{n}(q) \mapsto$ $\operatorname{End}\left(\left(\left(\mathbb{C}^{2}\right)^{\otimes n}\right)^{\otimes N}\right)$

$$
\Theta\left(\mathbb{U}_{l}\right)=\prod_{i=1}^{n} \rho_{a_{i}}\left(\mathbb{U}_{l^{(i)}}\right),
$$

where $a_{i}$ is the parameter associated with the $i$-th copy of the Temperley-Lieb algebra and is a representation of the Temperley-Lieb algebra itself provided that:

$$
(-1)^{n} \prod_{i=1}^{n}\left(a_{i}+a_{i}^{-1}\right)=-\left(q+q^{-1}\right) .
$$

The latter condition apparently follows from the quadratic relation (Hecke condition) of the Temperley-Lieb algebra. Now observe that the matrices $\rho_{a_{i}}\left(\mathbb{U}_{l^{(i)}}\right)$ commute among each other, 
since they act on different vector spaces. Having that in mind and taking into account that each one of the matrices satisfy the TL algebra, one can immediately show that $\Theta$ provides a representation of the TL algebra. Constraints are only entailed from the condition (4). One then obtains $n$ equations with $n$ unknown quantities (see Appendix A for more details on the Hecke condition), hence $a_{i}$ 's can be explicitly determined. Recall that the asymmetric twin representation involves two copies of the XXZ representation i.e. $n=2$. Note that we consider here the following sequence of spaces in the tensor product: $\cdots V_{l^{(1)}} \otimes V_{l^{(2)}} \otimes \cdots \otimes V_{l^{(n)}} \otimes V_{(l+1)^{(1)}} \otimes$ $V_{(l+1)^{(2)}} \otimes \cdots \otimes V_{(l+1)^{(n)}} \cdots, V \equiv \mathbb{C}^{2}$. It is clear that the total number of $\mathbb{C}^{2}$ spaces (i.e. total number of "sites") is $n N$.

The junction representation can be schematically represented as follows:

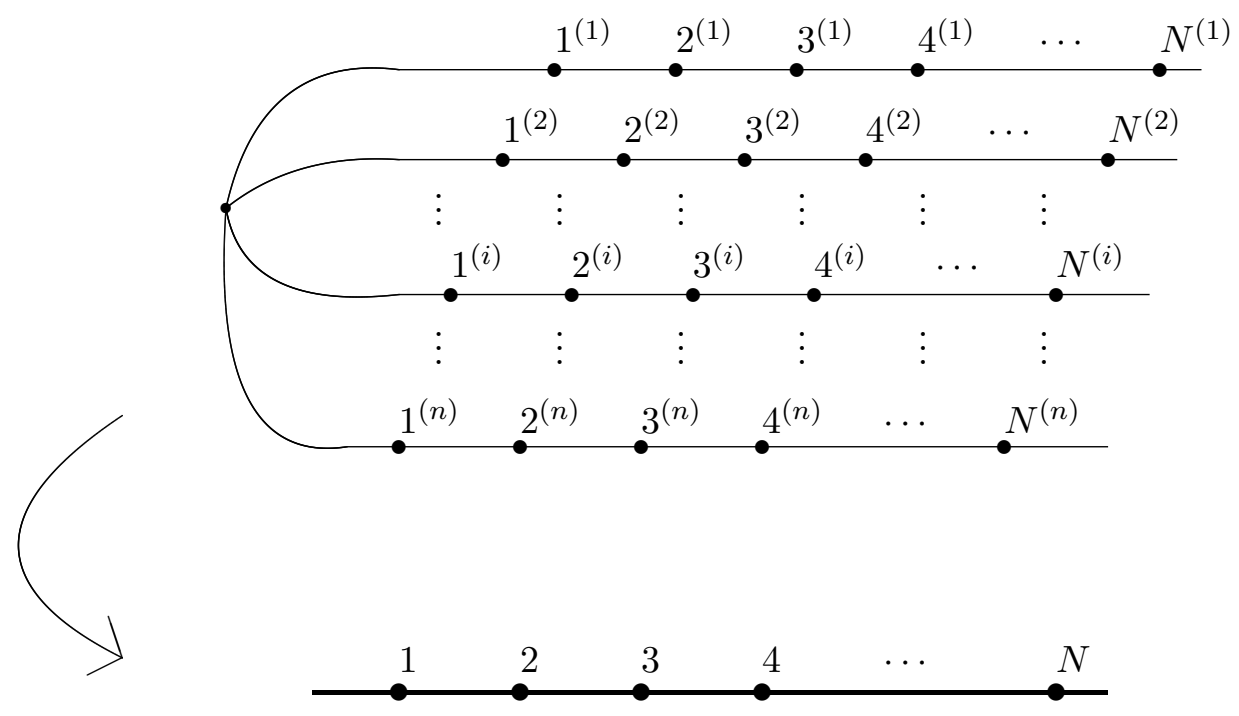

The thick line schematically presents the junction representation, showing somehow the "fusion" of $n$ copies of the XXZ representation. In particular, each one of the indices $l$ appearing there may be seen as a "fusion" of the indices $l^{(i)}$ as: $l \equiv\left\langle l^{(1)} l^{(2)} \ldots l^{(N)}\right\rangle, l+1 \equiv$ $\left\langle(l+1)^{(1)}(l+1)^{(2)} \ldots(l+1)^{(N)}\right\rangle$ and so on.

Let us now explicitly express each one of the terms in the product (3), defining the junction representation:

$$
\begin{aligned}
\rho_{a_{m}}\left(\mathbb{U}_{l^{(m)}}\right)=\cdots \mathbb{I} \otimes & (\sum_{a \neq b} \underbrace{\mathbb{I} \otimes \cdots \otimes \mathbb{I}}_{m-1} \otimes \underbrace{e_{a b}}_{l^{(m)}} \otimes \underbrace{\mathbb{I} \otimes \cdots \otimes \mathbb{I}}_{n-1} \otimes \underbrace{e_{b a}}_{(l+1)^{(m)}} \otimes \underbrace{\mathbb{I} \otimes \cdots \otimes \mathbb{I}}_{n-m} \\
& -\sum_{a \neq b} a_{m}^{-\operatorname{sgn}(a-b)} \underbrace{\mathbb{I} \otimes \cdots \otimes \mathbb{I}}_{m-1} \otimes \underbrace{e_{a a}}_{l^{(m)}} \otimes \underbrace{\mathbb{I} \otimes \cdots \otimes \mathbb{I}}_{n-1} \otimes \underbrace{e_{b b}}_{(l+1)^{(m)}} \otimes \underbrace{\mathbb{I} \otimes \cdots \otimes \mathbb{I}}_{n-m}) \otimes \mathbb{I} \cdots
\end{aligned}
$$

with $m \in\{1, \ldots, n\}$.

The matrix above clearly acts non-trivially on the spaces $\mathbb{C}_{l^{(m)}}^{2} \otimes \mathbb{C}_{(l+1)^{(m)}}^{2}$.

\section{$2.1 \quad$ The symmetry: $n=3$}

For the sake of simplicity and for illustrating purposes, we shall focus in this section on the simplest non-trivial case where $n=3$. Nevertheless, expressions on the symmetries associated to higher $n$ will be presented in the next subsection. 
The junction representation of the Temperley-Lieb algebra is in this case $\Theta: T_{N}(q) \mapsto$ $\operatorname{End}\left(\left(\mathbb{C}^{2} \otimes \mathbb{C}^{2} \otimes \mathbb{C}^{2}\right)^{\otimes N}\right)$

$$
\Theta\left(\mathbb{U}_{l}\right)=\rho_{a_{1}}\left(\mathbb{U}_{l^{(1)}}\right) \rho_{a_{2}}\left(\mathbb{U}_{l^{(2)}}\right) \rho_{a_{3}}\left(\mathbb{U}_{l^{(3)}}\right) .
$$

It is clear from their structure that the above matrices $\rho_{a_{i}}\left(\mathbb{U}_{l^{(i)}}\right)$ act on different spaces and hence they commute with each other. Let us now come to the study of the exact quantum symmetry algebras associated to the junction representation.

Let us first briefly recall the $U_{q}\left(s l_{2}\right)$ algebra, defined by generators $e, f, h$ that satisfy [13]:

$$
[h, e]=2 e, \quad[h, f]=-2 f, \quad[e, f]=\frac{q^{h}-q^{-h}}{q-q^{-1}} .
$$

The quantum algebra is as known equipped with a non-trivial co-product $\Delta: U_{q}\left(s l_{2}\right) \mapsto$ $U_{q}\left(s l_{2}\right) \otimes U_{q}\left(s l_{2}\right)$

$$
\Delta\left(q^{h}\right)=q^{h} \otimes q^{h}, \quad \Delta(x)=x \otimes q^{\frac{h}{2}}+q^{-\frac{h}{2}} \otimes x, \quad x \in\{e, f\} .
$$

The $n$ co-product may be obtained by iteration as: $\Delta^{(n)}=\left(\mathbb{I} \otimes \Delta^{(n-1)}\right) \Delta$.

Recall that the spin $\frac{1}{2}$ representation of the algebra above is given by, $\pi_{q}: U_{q}\left(s l_{2}\right) \mapsto \operatorname{End}\left(\mathbb{C}^{2}\right)$

$$
\pi_{q}(h)=\sigma^{z}, \quad \pi_{q}(e)=\sigma^{+}, \quad \pi_{q}(f)=\sigma^{-},
$$

where $\sigma^{z}, \sigma^{ \pm}$are the $2 \times 2$ Pauli matrices. Now define:

$$
\begin{aligned}
& \pi_{1}(x)=\pi_{a_{1}}(x) \otimes \mathbb{I} \otimes \mathbb{I}, \quad \pi_{2}(x)=\mathbb{I} \otimes \pi_{a_{2}}(x) \otimes \mathbb{I}, \\
& \pi_{3}(x)=\mathbb{I} \otimes \mathbb{I} \otimes \pi_{a_{3}}(x), \quad x \in U_{a_{i}}\left(s l_{2}\right) .
\end{aligned}
$$

Concerning the junction representation, it is easy to check that:

$$
\left[\Theta\left(\mathbb{U}_{l}\right), \pi_{i}^{\otimes N}\left(\Delta^{(N)}(x)\right)\right]=0, \quad x \in U_{a_{i}}\left(s l_{2}\right), \quad i=1,2,3 .
$$

It is obvious that each one of the representations acts on the spaces $V_{l^{(i)}}$ separately, that is

$$
\left[\rho_{a_{i}}\left(U_{l^{(i)}}\right), \pi_{j}^{\otimes N}\left(\Delta^{(N)}(x)\right)\right]=0, \quad i, j \in\{1,2,3\},
$$

which immediately leads to (5). Hence, the junction representation enjoys a manifest

$$
\mathcal{G}_{0} \equiv U_{a_{1}}\left(s l_{2}\right) \otimes U_{a_{2}}\left(s l_{2}\right) \otimes U_{a_{3}}\left(s l_{2}\right)
$$

symmetry, as is trivially expected by its construction.

However, as in the case of the twin asymmetric representation $[7,6]$, our main objective is to extract non trivial quantum algebra realizations that commute with the junction representation. Consider the following representations $\mathrm{f}_{i}: U_{q_{i}}\left(s l_{2}\right) \mapsto \operatorname{End}\left(\left(\mathbb{C}^{(2)}\right)^{\otimes 3}\right), i=0,1,2,3$

$$
\begin{aligned}
& \mathrm{f}_{0}(h)=e_{11} \otimes e_{11} \otimes e_{11}-e_{22} \otimes e_{22} \otimes e_{22}=h_{1}^{(0)}-h_{2}^{(0)}, \\
& \mathrm{f}_{0}(e)=e_{12} \otimes e_{12} \otimes e_{12}, \quad \mathrm{f}_{0}(f)=e_{21} \otimes e_{21} \otimes e_{21}, \\
& \mathrm{f}_{0}\left(q_{0}^{h}\right)=\mathbb{I}+\left(q_{0}-1\right) h_{1}^{(0)}+\left(q_{0}^{-1}-1\right) h_{2}^{(0)}, \\
& \mathrm{f}_{1}(h)=e_{22} \otimes e_{11} \otimes e_{11}-e_{11} \otimes e_{22} \otimes e_{22}=h_{1}^{(1)}-h_{2}^{(1)}, \\
& \mathrm{f}_{1}(e)=e_{21} \otimes e_{12} \otimes e_{12}, \quad \mathrm{f}_{1}(f)=e_{12} \otimes e_{21} \otimes e_{21}, \\
& \mathrm{f}_{1}\left(q_{1}^{h}\right)=\mathbb{I}+\left(q_{1}-1\right) h_{1}^{(1)}+\left(q_{1}^{-1}-1\right) h_{2}^{(1)}, \\
& \mathrm{f}_{2}(h)=e_{11} \otimes e_{22} \otimes e_{11}-e_{22} \otimes e_{11} \otimes e_{22}=h_{1}^{(2)}-h_{2}^{(2)},
\end{aligned}
$$




$$
\begin{aligned}
& \mathrm{f}_{2}(e)=e_{12} \otimes e_{21} \otimes e_{12}, \quad \mathrm{f}_{2}(f)=e_{21} \otimes e_{12} \otimes e_{21}, \\
& \mathrm{f}_{2}\left(q_{2}^{h}\right)=\mathbb{I}+\left(q_{2}-1\right) h_{1}^{(2)}+\left(q_{2}^{-1}-1\right) h_{2}^{(2)}, \\
& \mathrm{f}_{3}(h)=e_{11} \otimes e_{11} \otimes e_{22}-e_{22} \otimes e_{22} \otimes e_{11}=h_{1}^{(3)}-h_{2}^{(3)}, \\
& \mathrm{f}_{3}(e)=e_{12} \otimes e_{12} \otimes e_{21}, \quad \mathrm{f}_{3}(f)=e_{21} \otimes e_{21} \otimes e_{12}, \\
& \mathrm{f}_{3}\left(q_{3}^{h}\right)=\mathbb{I}+\left(q_{3}-1\right) h_{1}^{(3)}+\left(q_{3}^{-1}-1\right) h_{2}^{(3)} .
\end{aligned}
$$

It is straightforward to check that each one of the $f_{i}$ 's defined above provide a representation of $U_{q_{i}}\left(s l_{2}\right)$.

After some quite cumbersome but straightforward computation, it is shown that

$$
\left[\Theta\left(\mathbb{U}_{l}\right), \mathrm{f}_{i}^{\otimes N}\left(\Delta^{(N)}(x)\right)\right]=0, \quad x \in U_{q_{i}}\left(s l_{2}\right), \quad i=0,1,2,3,
$$

with $q_{i}$ to be determined by symmetry requirements. In Appendix B some explicit computations for $i=0$ are presented.

This in fact turns out to be an interesting combinatorics problem. Each symmetry is associated to a distinct $q_{i}$, which is determined by symmetry requirements. In particular, we found the following values for $q_{i}$, associated to the four representations $\mathrm{f}_{i}$ :

$$
q_{0}=a_{1} a_{2} a_{3}, \quad q_{1}=a_{1}^{-1} a_{2} a_{3}, \quad q_{2}=a_{1} a_{2}^{-1} a_{3} \quad q_{3}=a_{1} a_{2} a_{3}^{-1}
$$

(see also the Appendix A on the solution of the Hecke condition).

To summarize, this is the announced non trivial symmetry of the junction representation. For the simplest case, $n=3$, we have extracted the extra non-trivial symmetry of the junction representation, i.e.

$$
\mathcal{G} \equiv U_{q_{0}}\left(s l_{2}\right) \otimes U_{q_{1}}\left(s l_{2}\right) \otimes U_{q_{2}}\left(s l_{2}\right) \otimes U_{q_{3}}\left(s l_{2}\right)
$$

essentially different from the manifest $\bigotimes_{i=1}^{3} U_{a_{i}}\left(s l_{2}\right)$ symmetry. It is important to note that this extra symmetry is structurally much richer than the manifest one, and is enlarged almost exponentially with respect to $n$, as will become transparent in the subsequent section.

\subsection{Generic junction representation and exact symmetries}

We shall generalize here the investigation of the exact symmetries associated to the generic junction representation $(n>3)$. Inspired by the $n=3$ case, and the respective form of the representations that have been obtained there, we propose a big family of representations below for generic values of $n$. Actually, after inspection, one observes that the whole analysis merely reduces to a combinatorial problem.

Let us describe the generic family of representations of the quantum algebra. Consider first the representation $\mathrm{f}_{0}: U_{q_{0}}\left(s l_{2}\right) \mapsto \operatorname{End}\left(\left(\mathbb{C}^{(2)}\right)^{\otimes n}\right)$ as a starting point

$$
\begin{aligned}
& \mathrm{f}_{0}(h)=\left(e_{11}\right)^{\otimes n}-\left(e_{22}\right)^{\otimes n} \equiv h_{1}^{(0)}-h_{2}^{(0)}, \quad \mathrm{f}_{0}(e)=\left(e_{12}\right)^{\otimes n}, \quad \mathrm{f}_{0}(f)=\left(e_{21}\right)^{\otimes n}, \\
& \mathrm{f}_{0}\left(q_{0}^{h}\right)=\mathbb{I}+\left(q_{0}-1\right) h_{1}^{(0)}+\left(q_{0}^{-1}-1\right) h_{2}^{(0)} .
\end{aligned}
$$

Recall that in the generic situation, $n$ constants $a_{i}$ are involved. The symmetry algebras examined here are in fact associated to all possible combinations of $a_{i}$ 's and their inverses. More precisely, consider first the combination $a_{1} a_{2} \cdots a_{n}$, which is in fact equal to $q_{0}$. By turning one of the $a_{i}$ to its inverse we obtain $n$ such combinations. Then we may turn two, three $a_{i}$ 's etc. into their inverses, and stop turning after we reach the values $\frac{n}{2}$ or $\frac{n-1}{2}$ (for $n$ even and odd respectively). We stop basically because we reproduce the same algebras as we shall see 
below. In fact, by repeating the procedure above we end up with a total number of combinations given by:

$$
\begin{aligned}
& M=\sum_{m=0}^{\frac{n-1}{2}} \frac{n !}{m !(n-m) !} \quad \text { if } n \text { odd, } \\
& M=\sum_{m=0}^{\frac{n}{2}-1} \frac{n !}{m !(n-m) !}+\frac{1}{2} \frac{n !}{\frac{n}{2} ! \frac{n}{2} !} \quad \text { if } n \text { even. }
\end{aligned}
$$

We can now introduce the following generic family of representations $\mathrm{f}_{i_{1} i_{2} \ldots i_{m}}, m \in\{0, \ldots, L\}$ where $L=\frac{n}{2}$ for $n$ even and $L=\frac{n-1}{2}$ for $n$ odd (for $m=0$ we basically obtain $\mathrm{f}_{0}$ ). The corresponding deformation parameter is defined as

$$
q_{i_{1} i_{2} \ldots i_{m}}=(-1)^{n+1} a_{1} a_{2} \cdots a_{i_{1}}^{-1} a_{i_{1}+1} \cdots a_{i_{2}}^{-1} \cdots a_{i_{m}}^{-1} \cdots a_{n},
$$

that is each one of the constants $a_{i_{j}}, i_{j} \in\{1, \ldots, n\}$ is turned to its inverse. It is thus clear that the total number of representations is given by the total number of combinations $M$ given in (8). In fact, for every $m$ we have $\frac{n !}{m !(n-m) !}$ number of representations, provided that for $m=0$ there is only one representation, the $\mathrm{f}_{0}$. Note also that

$$
q_{i_{1} \ldots i_{m}}=q_{\mathbb{P}\left(i_{1} \ldots i_{m}\right)},
$$

where $\mathbb{P}$ denotes all possible permutations among the elements $i_{j}$. Notice that in the case of $n$ even and $m=\frac{n}{2}$ we keep only half of the combinations. For instance take $n=4$; we should only consider $q_{34}, q_{24}, q_{23}$. It is clear that $q_{12}=q_{34}^{-1}, \mathrm{f}_{12}(e)=\mathrm{f}_{34}(f), \mathrm{f}_{12}(f)=\mathrm{f}_{34}(e)$, and similarly for the rest (see also Appendix $\mathrm{C}$ for a more detailed description of the algebra elements). More generally, let us introduce the conjugate index:

$$
\bar{i}=n-i+1 \text {. }
$$

It is then clear that

$$
q_{i_{1} \ldots i \frac{n}{2}}=q_{\bar{i}_{1} \ldots \bar{i}_{\frac{n}{2}}}^{-1} .
$$

It is worth noting that some kind of duality is manifest throughout this process. In particular, the quantum algebra with parameter $q_{i_{1} \ldots i_{m}}$ is equivalent to the algebra with parameter $q_{i_{1} \ldots i_{m}}^{-1}$, after interchanging $e \leftrightarrow f$ and $h \leftrightarrow-h$. This is the reason why we stop the procedure of turning $a_{i}$ 's to their inverses after reaching the values $\frac{n}{2}$ or $\frac{n-1}{2}$ for $n$ even and odd respectively, as described earlier in the text. Consequently, physical systems which may possess such underlying structures are expected to enjoy some kind of physical equivalence that reflect this duality.

A generic representation is then defined along the lines described above as

$$
\begin{aligned}
& \mathrm{f}_{i_{1} i_{2} \ldots i_{m}}: U_{q_{i_{1} i_{2} \ldots i_{m}}}\left(s l_{2}\right) \rightarrow \operatorname{End}\left(\left(\mathbb{C}^{2}\right)^{\otimes n}\right), \\
& \begin{aligned}
\mathrm{f}_{i_{1} i_{2} \ldots i_{m}}(h)= & e_{11} \otimes \cdots e_{11} \otimes \cdots \otimes \underbrace{e_{22}}_{i_{1}} \otimes e_{11} \cdots \otimes \underbrace{e_{22}}_{i_{2}} \otimes \cdots \otimes \underbrace{e_{22}}_{i_{m}} \otimes \cdots \otimes e_{11} \\
& \quad-e_{22} \otimes \cdots e_{22} \otimes \cdots \otimes \underbrace{e_{11}}_{i_{1}} \otimes e_{22} \cdots \otimes \underbrace{e_{11}}_{i_{2}} \otimes \cdots \otimes \underbrace{e_{11}}_{i_{m}} \otimes \cdots \otimes e_{22} \\
\equiv & h_{1}^{\left(i_{1} \ldots i_{m}\right)}-h_{2}^{\left(i_{1} \ldots i_{m}\right)} \\
\mathrm{f}_{i_{1} i_{2} \ldots i_{m}}(e)= & e_{12} \otimes \cdots e_{12} \otimes \cdots \otimes \underbrace{e_{21}}_{i_{1}} \otimes e_{12} \cdots \otimes \underbrace{e_{21}}_{i_{2}} \otimes \cdots \otimes \underbrace{e_{21}}_{i_{m}} \otimes \cdots \otimes e_{12},
\end{aligned}
\end{aligned}
$$




$$
\mathrm{f}_{i_{1} i_{2} \ldots i_{m}}(f)=e_{21} \otimes \cdots e_{21} \otimes \cdots \otimes \underbrace{e_{12}}_{i_{1}} \otimes e_{21} \cdots \otimes \underbrace{e_{12}}_{i_{2}} \otimes \cdots \otimes \underbrace{e_{12}}_{i_{m}} \otimes \cdots \otimes e_{21} .
$$

All the above quantities emerge from $f_{0}$ by exchanging:

$$
e_{11} \leftrightarrow e_{22} \quad \text { for } h, \quad e_{12} \leftrightarrow e_{21} \quad \text { for } e, f,
$$

for every site $i_{j} \in\{1,2, \ldots, n\}$.

In a similar fashion the generalized representation for the $q^{h}$ element has the form

$$
\mathrm{f}_{q_{i_{1} \ldots i_{m}}}\left(q_{i_{1} \ldots i_{m}}^{h}\right)=\mathbb{I}+\left(q_{i_{1} \ldots i_{m}}-1\right) h_{1}^{\left(i_{1} \ldots i_{m}\right)}+\left(q_{i_{1} \ldots i_{m}}^{-1}-1\right) h_{2}^{\left(i_{1} \ldots i_{m}\right)} .
$$

To prove that the above relations define a representation of the $U_{q_{i_{1} \ldots i i_{m}}}\left(s l_{2}\right)$ algebra is a straightforward task. One just takes into account the basic property: $e_{i j} e_{k l}=\delta_{j k} e_{i l}$.

Having obtained this big family of $U_{q_{i_{1} \ldots i_{m}}}\left(s l_{2}\right)$ representations, we conjecture that these are also non-trivial exact symmetries of the junction representation. More precisely, our main conjecture is that:

$$
\left[\mathrm{f}_{i_{1} \ldots i_{m}}^{\otimes N}\left(\Delta^{(N)}(x)\right), \Theta\left(\mathbb{U}_{l}\right)\right]=0, \quad x \in U_{q_{i_{1} \ldots i_{m}}}\left(s l_{2}\right) .
$$

This is a plausible conjecture given the results for $n=3$ and the particular structure of the mentioned representations. Note that we have also checked the validity of our conjecture for the cases $n=4,5$ with the use of the algebraic program Mathematica.

Let us make some general qualitative comments that further enforce our claim. Consider first the multiplication ${ }^{1} \Theta\left(\mathbb{U}_{1}\right) \mathrm{f}_{0}^{\otimes 2}(\Delta(h))$. Note that $\Theta$ contains a large number of different terms inside, which can be grouped into powers of $a$ 's. (From now on and only for the following argument we let $a_{i}=a y_{i}$ and count in powers of $a$.) Actually the number of terms of a specific power $\mathcal{O}\left(a^{m}\right)$ is given in terms of the binomial coefficient, but this is not so significant at the moment. On the other hand, it is instructive to note that the terms containing $a$ 's are either $e_{11}$ or $e_{22}$, while those not containing $a$ 's are $e_{12}$ and $e_{21}$.

Consider now the multiplication of $\Theta$ with the first term of $\mathrm{f}_{0}^{\otimes 2}(\Delta(h))$, that is with

$$
\left(e_{11}\right)^{\otimes n} \otimes \mathbb{I}^{\otimes n} .
$$

Recall that $\Theta$ is naturally "broken" into two parts, each one living on $\left(\mathbb{C}^{2}\right)^{\otimes n}$. We see that in order for the product of $\Theta$ with the above term to be different from zero, the first part of $\Theta$ should only contain the basis vectors $e_{11}$ and $e_{21}$, in every possible combination. Otherwise it just gives zero. Consequently, the problem is reduced into a combinatorics problem. However, for the case of $\mathrm{f}_{0}^{\otimes 2}(\Delta(h))$ the situation is easy to handle ${ }^{2}$, and we see that the product $\Theta\left(\mathbb{U}_{1}\right) \mathrm{f}_{0}^{\otimes 2}(\Delta(h))$ vanishes identically. The reason is the following.

We observe that from the multiplication of $\Theta$ with the first term of $\mathrm{f}_{0}^{\otimes 2}(\Delta(h))$ only some specific terms survive. However, these are exactly the same terms that survive the multiplication of $\Theta$ with the last term of the co-product, namely with

$$
\mathbb{I}^{\otimes n} \otimes\left(e_{22}\right)^{\otimes n}
$$

And since those two terms appear with a different sign, they cancel out. The same happens with the other two terms. The same argument is also valid for the second term of the commutator,

\footnotetext{
${ }^{1}$ We focus here on the $f_{0}$ representation but our arguments may be applied for the generic family of representations.

${ }^{2}$ For the co-products of the other two elements the situation is more difficult to handle, since the products with $\Theta$ do not vanish identically. Hence the various conditions relating $a_{i}$ 's must be taken into account, which makes the presentation of a general argument a difficult task.
} 
that is for the multiplication $\mathrm{f}_{0}^{\otimes 2}(\Delta(h)) \Theta$, which is identically zero. Hence, we have shown using a somehow heuristic argument - that

$$
\left[\Theta\left(\mathbb{U}_{1}\right), \mathrm{f}_{0}^{\otimes 2}(\Delta(h))\right]=0 .
$$

Note that this result is valid for all the representations that belong to the generic family that we have considered so far, due to the highly symmetric nature of $\mathrm{f}_{i_{1} \ldots i_{m}}^{\otimes 2}(\Delta(h))$. It should be stressed that although the above argument is not a rigorous proof, we expect that it holds for the whole family of representations. Similar arguments, albeit more complicated in their technicalities, can be presented for the other two elements of the quantum algebra.

Finally, let us briefly discuss for completeness the existence of trivial symmetries associated to the junction representation. Define:

$$
\pi_{i}(x)=\mathbb{I} \otimes \cdots \otimes \cdots \underbrace{\pi_{a_{i}}(x)}_{1^{(i)}} \otimes \cdots \otimes \mathbb{I}, \quad x \in U_{a_{i}}\left(s l_{2}\right) .
$$

It is straightforward then to check that the junction representation commutes with the actions defined above. Each one of the representations $\rho_{a_{i}}$ acts non trivially on the spaces $\mathbb{C}_{l^{(i)}}^{2} \otimes \mathbb{C}_{(l+1)^{(i)}}^{2}$ separately, that is

$$
\left[\rho_{a_{i}}\left(U_{l^{(i)}}\right), \pi_{j}^{\otimes N}\left(\Delta^{(N)}(x)\right)\right]=0, \quad i, j \in\{1, \ldots, n\},
$$

which immediately leads to

$$
\left[\Theta\left(\mathbb{U}_{l}\right), \pi_{i}^{\otimes N}\left(\Delta^{(N)}(x)\right)\right]=0, \quad x \in U_{a_{i}}\left(s l_{2}\right),
$$

exposing the manifest symmetry of a generic junction representation

$$
\mathcal{G}_{0} \equiv U_{a_{1}}\left(s l_{2}\right) \otimes U_{a_{2}}\left(s l_{2}\right) \otimes \cdots \otimes U_{a_{n}}\left(s l_{2}\right)
$$

To summarize, we have been able to explicitly prove the existence of non-trivial exact symmetries (6), (7) associated to the junction representation for $n=3$ (see Appendix B). We have conjectured the generic form of non-trivial symmetries of the junction representation (9) $\forall n$, and we have checked the validity of our conjecture (10) with Mathematica for $n=4,5$. The existence of trivial symmetries (11) is an immediate consequence of the structure of the junction representation, therefore the proof of (12), (13) is straightforward $\forall n$.

\section{Boundary Temperley-Lieb algebra and representations}

We shall discuss in this section extensions of the junction representation in the case of the boundary Temperley-Lieb algebra. The boundary Temperley-Lieb $B_{N}(q, Q)$ algebra is defined by generators $\mathbb{U}_{i}$ obeying (1) and $\mathbb{U}_{0}$ satisfying

$$
\mathbb{U}_{0}^{2}=\delta_{0} \mathbb{U}_{0}, \quad \mathbb{U}_{1} \mathbb{U}_{0} \mathbb{U}_{1}=\kappa \mathbb{U}_{1}, \quad\left[\mathbb{U}_{0}, \mathbb{U}_{i}\right]=0, \quad i>1,
$$

where $\delta_{0}=-\left(Q+Q^{-1}\right), \kappa=q Q^{-1}+q^{-1} Q$, although there is a reparametrization freedom that one can appropriately use (see e.g. [7]).

The well known XXZ representation of the boundary Temperley-Lieb algebra is given next. Let $U$ be the matrix defined in (2) and

$$
U_{0}=-Q^{-1} e_{11}-Q e_{22}+e_{12}+e_{21}
$$


Then the following is a representation of the boundary TL algebra,

$$
\begin{aligned}
& \rho_{q, Q}: \quad B_{N}(q, Q) \mapsto \operatorname{End}\left(\left(\mathbb{C}^{2}\right)^{\otimes N}\right), \\
& \rho_{q, Q}\left(\mathbb{U}_{i}\right)=\mathbb{I} \otimes \cdots \otimes \mathbb{I} \otimes \underbrace{U}_{i, i+1} \otimes \mathbb{I} \otimes \cdots \otimes \mathbb{I}, \quad \rho_{q, Q}\left(\mathbb{U}_{0}\right)=U_{0} \otimes \mathbb{I} \otimes \cdots \otimes \mathbb{I} .
\end{aligned}
$$

The obvious junction representation for the "boundary" element for generic $n$ is then the following

$$
\Theta\left(\mathbb{U}_{0}\right)=\prod_{i=1}^{n} \rho_{a_{i}, Q_{i}}\left(\mathbb{U}_{0}\right),
$$

where each one of the XXZ representations act on the spaces $\mathbb{C}_{1^{(1)}}^{2}, \mathbb{C}_{1^{(2)}}^{2}, \ldots, \mathbb{C}_{1^{(n)}}^{2}$; i.e.

$$
\rho_{a_{i}, Q_{i}}\left(\mathbb{U}_{0}\right)=\mathbb{I} \otimes \cdots \otimes \underbrace{U_{0}}_{1^{(i)}} \otimes \cdots \otimes \mathbb{I}
$$

It is straightforward to check, due to the fact that spaces $\mathbb{C}_{l^{(i)}}^{2}$ commute among each other that $\Theta\left(\mathbb{U}_{i}\right)$ and $\Theta\left(\mathbb{U}_{0}\right)$ do satisfy the algebraic relations of the boundary TL algebra, thus they provide a representation of the algebra.

However, our objective here is to search for non trivial boundary elements that mix essentially the spaces exactly as the non trivial symmetries mix the various spaces $\mathbb{C}_{1^{(i)}}^{2}$. Consider the following matrices

$$
M_{i_{1} \ldots i_{m}}=-Q^{-1} h_{1}^{\left(i_{1} \ldots i_{m}\right)}-Q h_{2}^{\left(i_{1} \ldots i_{m}\right)}+\mathrm{f}_{i_{1} \ldots i_{m}}(e)+\mathrm{f}_{i_{1} \ldots i_{m}}(f)
$$

and recall that $h_{1,2}^{\left(i_{1} \ldots i_{m}\right)}$ and $\mathrm{f}_{i_{1} \ldots i_{m}}(e, f)$ are defined in Section 2.2. The indices take values $i_{j} \in\{1, \ldots, n\}$ as also described in Section 2.2.

Let us focus again on the $n=3$ case, where analytic proofs of our claims are available (for higher $n$ analytic proofs are highly complicated, and only checks with the help of algebraic packages can be performed). First, introduce the following matrices corresponding to the four $\mathrm{f}_{i}$ representations of the $n=3$ case described in Section 2.1,

$$
\begin{aligned}
& M_{0}=-Q^{-1} e_{11} \otimes e_{11} \otimes e_{11}-Q e_{22} \otimes e_{22} \otimes e_{22}+e_{12} \otimes e_{12} \otimes e_{12}+e_{21} \otimes e_{21} \otimes e_{21}, \\
& M_{1}=-Q^{-1} e_{22} \otimes e_{11} \otimes e_{11}-Q e_{11} \otimes e_{22} \otimes e_{22}+e_{21} \otimes e_{12} \otimes e_{12}+e_{12} \otimes e_{21} \otimes e_{21}, \\
& M_{2}=-Q^{-1} e_{11} \otimes e_{22} \otimes e_{11}-Q e_{22} \otimes e_{11} \otimes e_{22}+e_{12} \otimes e_{21} \otimes e_{12}+e_{21} \otimes e_{12} \otimes e_{21}, \\
& M_{3}=-Q^{-1} e_{11} \otimes e_{11} \otimes e_{22}-Q e_{22} \otimes e_{22} \otimes e_{11}+e_{12} \otimes e_{12} \otimes e_{21}+e_{21} \otimes e_{21} \otimes e_{12} .
\end{aligned}
$$

The matrices $M_{i}$ act on $\mathbb{C}_{1^{(1)}}^{2} \otimes \mathbb{C}_{1^{(2)}}^{2} \otimes \mathbb{C}_{1^{(3)}}^{2}$. Also set

$$
\mathcal{M}_{i}=M_{i} \otimes \mathbb{I} \otimes \cdots \otimes \mathbb{I}
$$

where now $\mathbb{I}$ acts on $\left(\mathbb{C}^{2}\right)^{\otimes 3}$. It can be shown then that

$$
\Theta\left(\mathbb{U}_{1}\right) \mathcal{M}_{i} \Theta\left(\mathbb{U}_{1}\right)=\kappa \Theta\left(\mathbb{U}_{1}\right), \quad i=0,1,2,3,
$$

and this suffices to prove that this is a representation of the boundary Temperley-Lieb algebra. Indeed we define the representation $\Theta_{i}: B_{N}(q, Q) \mapsto \operatorname{End}\left(\mathbb{C}^{2} \otimes \mathbb{C}^{2} \otimes \mathbb{C}^{2}\right)^{\otimes N}, i \in\{0,1,2,3\}$ such that:

$$
\Theta_{i}\left(\mathbb{U}_{l}\right)=\Theta\left(\mathbb{U}_{l}\right), \quad l \in\{1,2, \ldots, N-1\}, \quad \Theta_{i}\left(\mathbb{U}_{0}\right)=\mathcal{M}_{i}
$$


We can proceed then and state the following generic conjecture $\forall n>3$. We conjecture that $\Theta_{i_{1} \ldots i_{m}}$ such that

$$
\begin{aligned}
& \Theta_{i_{1} \ldots i_{m}}\left(\mathbb{U}_{l}\right)=\Theta\left(\mathbb{U}_{l}\right), \quad l \in\{1, \ldots, N-1\}, \quad \Theta_{i_{1} \ldots i_{m}}\left(\mathbb{U}_{0}\right)=\mathcal{M}_{i_{1} \ldots i_{m}}, \\
& \mathcal{M}_{i_{1} \ldots i_{m}}=M_{i_{1} \ldots i_{m}} \otimes \mathbb{I} \otimes \cdots \otimes \mathbb{I}
\end{aligned}
$$

is a representation of the boundary Temperley-Lieb algebra. To prove this it is sufficient to show that

$$
\Theta\left(\mathbb{U}_{1}\right) \mathcal{M}_{i_{1} \ldots i_{m}} \Theta\left(\mathbb{U}_{1}\right)=\kappa \Theta\left(\mathbb{U}_{1}\right) .
$$

As before, we have checked the validity of the latter conjecture by performing an analytical computation for $n=3$ and by using Mathematica for the cases $n=4,5$. Therefore, we have strong indications that it is valid for generic $n$. The analytic proof for $n=3$ is omitted here for brevity, but it is straightforward, although technically demanding, and is based on the explicit form of $\Theta\left(\mathbb{U}_{1}\right), \mathcal{M}_{i_{1} \ldots i_{m}}$ and the property: $e_{i j} e_{k l}=\delta_{j k} e_{i l}$

As mentioned above, the structure of these boundary elements is inspired by the form of the non trivial symmetries studied in the previous section. It will be transparent in the subsequent section that they break the respective symmetries then, along the lines described in $[7,8,6]$.

\subsection{Residual symmetries}

In this section we shall examine how each one of the non-trivial boundary elements $\mathcal{M}_{i_{1} \ldots i_{m}}$ breaks the original symmetry of the Temperley-Lieb algebra. This will be particularly useful when studying the corresponding quantum spin chain symmetry.

Apart from the manifest symmetry of the algebra, we have exposed in the previous section a $U_{q_{0}}\left(s l_{2}\right) \otimes U_{q_{1}}\left(s l_{2}\right) \otimes U_{q_{2}}\left(s l_{2}\right) \otimes U_{q_{3}}\left(s l_{2}\right)$ symmetry for the junction representation. It is straightforward to check that each one of the non-trivial boundary elements breaks only part of this symmetry, while preserving the rest of it. In particular, it is shown that

$$
\left[\mathrm{f}_{i}(x), M_{j}\right]=0, \quad x \in U_{q_{i}}\left(s l_{2}\right), \quad i \neq j .
$$

The above condition is not accidental at all. In fact, it is somehow expected, given that the structure of those boundary elements is strictly related to the respective non-trivial symmetries of the algebra. However, the case where $i=j$ requires particular attention.

Let us first consider the following combination of generators of the quantum algebra $U_{q_{i_{1} \ldots i_{m}}}\left(s l_{2}\right)$ (see also e.g. $\left.[6,19,5]\right)$

$$
\mathcal{Q}_{i_{1} \ldots i_{m}}=q_{i_{1} \ldots i_{m}}^{-\frac{1}{2}} q_{i_{1} \ldots i_{m}}^{\frac{h}{2}} e+q_{i_{1} \ldots i_{m}}^{\frac{1}{2}} q_{i_{1} \ldots i_{m}}^{-\frac{h}{2}} f+x_{i_{1} \ldots i_{m}} q_{i_{1} \ldots i_{m}}^{h}-x_{i_{1} \ldots i_{m}} \mathbb{I}
$$

where $x_{i_{1} \ldots i_{m}}$ are constants to be determined later on. Focus again on the simplest non-trivial situation $n=3$. Using then the four $\mathrm{f}_{i}$ representations at hand one may compute the respective charges, which have the following form

$$
\begin{aligned}
\mathrm{f}_{0}\left(\mathcal{Q}_{0}\right)= & \left(-x_{0}+q_{0} x_{0}\right) e_{11} \otimes e_{11} \otimes e_{11}+e_{12} \otimes e_{12} \otimes e_{12} \\
& +e_{21} \otimes e_{21} \otimes e_{21}+\left(-x_{0}+q_{0}^{-1} x_{0}\right) e_{22} \otimes e_{22} \otimes e_{22}, \\
\mathrm{f}_{1}\left(\mathcal{Q}_{1}\right)= & \left(-x_{1}+q_{1} x_{1}\right) e_{22} \otimes e_{11} \otimes e_{11}+e_{21} \otimes e_{12} \otimes e_{12} \\
& +e_{12} \otimes e_{21} \otimes e_{21}+\left(-x_{1}+q_{1}^{-1} x_{1}\right) e_{11} \otimes e_{22} \otimes e_{22}, \\
\mathrm{f}_{2}\left(\mathcal{Q}_{2}\right)= & \left(-x_{2}+q_{2} x_{2}\right) e_{11} \otimes e_{22} \otimes e_{11}+e_{12} \otimes e_{21} \otimes e_{12} \\
& +e_{21} \otimes e_{12} \otimes e_{21}+\left(-x_{2}+q_{2}^{-1} x_{2}\right) e_{22} \otimes e_{11} \otimes e_{22},
\end{aligned}
$$




$$
\begin{aligned}
f_{3}\left(\mathcal{Q}_{3}\right)= & \left(-x_{3}+q_{3} x_{3}\right) e_{11} \otimes e_{11} \otimes e_{22}+e_{12} \otimes e_{12} \otimes e_{21} \\
& +e_{21} \otimes e_{21} \otimes e_{12}+\left(-x_{3}+q_{3}^{-1} x_{3}\right) e_{22} \otimes e_{22} \otimes e_{11} .
\end{aligned}
$$

This charge commutes with all the irrespective boundary elements, that is

$$
\left[\mathrm{f}_{i}\left(\mathcal{Q}_{i}\right), M_{j}\right]=0 \quad \text { for } i \neq j,
$$

but is also found to commute with the relevant boundary element

$$
\left[\mathrm{f}_{i}\left(\mathcal{Q}_{i}\right), M_{i}\right]=0,
$$

provided that the constants $x_{i}$ satisfy

$$
x_{i}=\frac{Q-Q^{-1}}{q_{i}-q_{i}^{-1}} .
$$

To conclude, the symmetry we extract here for each representation $\Theta_{i}$ of the boundary Temperley-Lieb algebra is:

$$
\left[\Theta_{i}\left(\mathbb{U}_{l}\right), \mathrm{f}_{i}^{\otimes N}\left(\Delta^{(N)}\left(\mathcal{Q}_{i}\right)\right) \otimes_{i \neq j} \mathrm{f}_{j}^{\otimes N}\left(\Delta^{(N)}(x)\right)\right]=0, \quad x \in U_{q_{j}}\left(s l_{2}\right) .
$$

We may generalize the statement - as a conjecture - on the boundary symmetry $\forall n$, i.e., we conjecture that

$$
\begin{aligned}
& {\left[\Theta_{i_{1} \ldots i_{m}}\left(\mathbb{U}_{l}\right), \mathrm{f}_{i_{1} \ldots i_{m}}^{\otimes N}\left(\Delta^{(N)}\left(\mathcal{Q}_{i_{1} \ldots i_{m}}\right)\right) \otimes_{\left\{i_{1}, \ldots, i_{m}\right\} \neq\left\{j_{1}, \ldots, j_{m}\right\}} \mathrm{f}_{j_{1} \ldots j_{m}}^{\otimes N}\left(\Delta^{(N)}(x)\right)\right]=0,} \\
& \quad x \in U_{q_{j_{1} \ldots j_{m}}}\left(s l_{2}\right) .
\end{aligned}
$$

It is clear that the trivial representation defined in (14), (15) enjoys the symmetry (see also [6]):

$$
\left[\Theta_{i}\left(\mathbb{U}_{l}\right), \pi_{i}^{\otimes N}\left(\Delta^{(N)}\left(\mathcal{Q}_{i}\right)\right) \otimes_{i \neq j} \pi_{j}^{\otimes N}\left(\Delta^{(N)}(x)\right)\right]=0, \quad x \in U_{a_{j}}\left(s l_{2}\right), \quad i, j \in\{1, \ldots, n\},
$$

where we define

$$
\mathcal{Q}_{i}=a_{i}^{-\frac{1}{2}} a_{i}^{\frac{h}{2}} e+a_{i}^{\frac{1}{2}} a_{i}^{-\frac{h}{2}} f+x_{i} a_{i}^{h}-x_{i} \mathbb{I}, \quad i \in\{1, \ldots, n\},
$$

and

$$
x_{i}=\frac{Q-Q^{-1}}{a_{i}-a_{i}^{-1}} .
$$

For a more detailed discussion on boundary symmetries we refer the interested reader to $[7,6]$.

\section{The quantum spin chain}

We shall now briefly discuss the corresponding quantum spin chain, enjoying the symmetries described above. Recall the $\check{R}$ matrix is a solution of the braid Yang-Baxter equation

$$
\check{R}_{12}\left(\lambda_{1}-\lambda_{2}\right) \check{R}_{23}\left(\lambda_{1}\right) \check{R}_{12}\left(\lambda_{2}\right)=\check{R}_{23}\left(\lambda_{2}\right) \check{R}_{12}\left(\lambda_{1}\right) \check{R}_{23}\left(\lambda_{1}-\lambda_{2}\right) .
$$

The $\check{R}$ matrix associated with representations of the Temperley-Lieb algebra may be expressed as $[12]$

$$
\check{R}_{i i+1}(\lambda)=\sinh (\lambda+i \mu)+\sinh \lambda \rho\left(\mathbb{U}_{i}\right),
$$

for any representation $\rho$ of the TL algebra $T_{N}(q)$, and where $q=e^{i \mu}$. 
Define the $R$ matrix as: $R(\lambda)=\mathcal{P} \check{R}(\lambda)$. The $R$ matrix associated with the junction representation satisfies unitarity and crossing symmetry i.e.

$$
R_{i j}(\lambda) R_{j i}(-\lambda) \propto \mathbb{I}, \quad R_{i j}(\lambda) \propto \mathbb{V}_{i} R_{i j}^{t_{j}}(-\lambda-i) \mathbb{V}_{i},
$$

where we define in general

$$
\mathbb{V}=\otimes_{i=1}^{n}\left(\begin{array}{cc} 
& a_{i}^{-\frac{1}{2}} \\
a_{i}^{\frac{1}{2}} &
\end{array}\right)
$$

and for $n=3$ in particular we have:

$$
\begin{aligned}
& \mathbb{V}=\left(\begin{array}{cc}
a^{\frac{1}{2}} & a_{1}^{-\frac{1}{2}} \\
a_{1}^{\frac{1}{2}}
\end{array}\right) \otimes\left(\begin{array}{cc}
a_{2}^{\frac{1}{2}} & a_{2}^{-\frac{1}{2}} \\
a_{2} &
\end{array}\right) \otimes\left(\begin{array}{cc} 
& a_{3}^{-\frac{1}{2}} \\
a_{3}^{\frac{1}{2}}
\end{array}\right.
\end{aligned}
$$

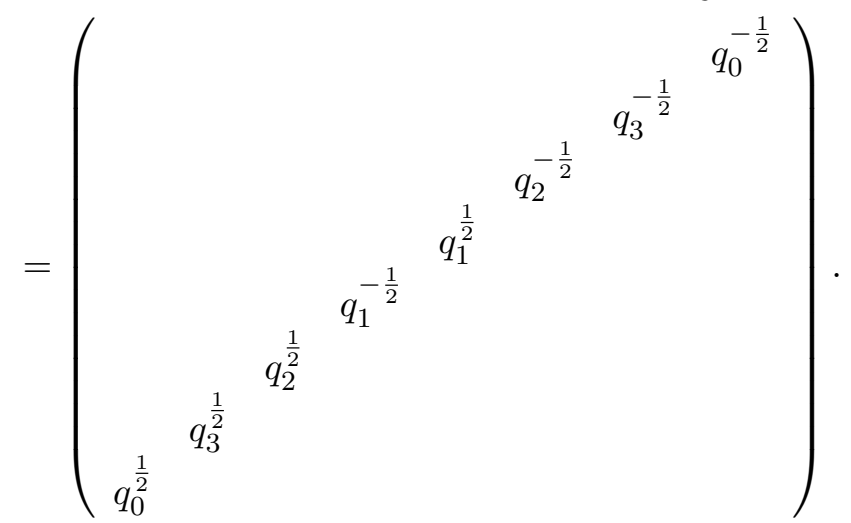

Recall also the identifications of $q_{i}$ 's, entailed by the exact symmetries of the representation.

Then one may construct the transfer matrix of an open spin chain with generic integrable boundary conditions [23]:

$$
t(\lambda)=\operatorname{tr}_{0}\left\{M_{0} K_{0}^{+} \mathbb{T}(\lambda)\right\}
$$

where

$$
\mathbb{T}=T_{0}(\lambda) K_{0}(\lambda) T_{0}^{-1}(\lambda), \quad T(\lambda)=R_{0 N}(\lambda) \cdots R_{01}(\lambda) .
$$

The $K$ matrix satisfies the reflection equation

$$
R_{12}\left(\lambda_{1}-\lambda_{2}\right) K_{1}\left(\lambda_{1}\right) R_{21}\left(\lambda_{1}+\lambda_{2}\right) K_{2}\left(\lambda_{2}\right)=K_{2}\left(\lambda_{2}\right) R_{12}\left(\lambda_{1}+\lambda_{2}\right) K_{1}\left(\lambda_{1}\right) R_{21}\left(\lambda_{1}-\lambda_{2}\right) .
$$

We shall focus here on the case of trivial left boundary, that is $K^{+} \propto \mathbb{I}$. Also $M$ is defined as

$$
M=\mathbb{V}^{t} \mathbb{V}
$$

and for $n=3$ in particular it becomes:

$$
M=\left(\begin{array}{cccccccc}
q_{0} & & & & & & & \\
& q_{3} & & & & & & \\
& & q_{2} & & & & & \\
& & & q_{1}^{-1} & & & & \\
& & & & q_{1} & & & \\
& & & & & q_{2}^{-1} & & \\
& & & & & & q_{3}^{-1} & \\
& & & & & & & q_{0}^{-1}
\end{array}\right) .
$$


It is known that solutions of the reflection equation may be expressed in terms of representations of the boundary Temperley-Lieb algebra as (see also e.g. [16, 7]):

$$
\begin{aligned}
& K(\lambda)=x(\lambda)+y(\lambda) \rho\left(\mathbb{U}_{0}\right), \\
& x(\lambda)=-\delta_{0} \cosh (2 \lambda+i \mu)-\kappa \cosh (2 \lambda)-\cosh (2 i \zeta), \\
& y(\lambda)=2 \sinh (2 \lambda) \sinh (i \mu) .
\end{aligned}
$$

Let us first consider the case of a trivial right boundary i.e. $K \propto \mathbb{I}$. Then as was proven in Section 2.1 that the junction representation enjoys the $\otimes_{i=0}^{3} U_{q_{i}}\left(s l_{2}\right)$ symmetry and consequently

$$
\left[\check{R}, \mathrm{f}_{i}^{\otimes 2}\left(\Delta^{\otimes 2}(x)\right)\right]=0 \quad \Rightarrow \quad \mathrm{f}_{i}^{\otimes 2}\left(\Delta^{\prime} \otimes 2(x)\right) R(\lambda)=R(\lambda) \mathrm{f}_{i}^{\otimes 2}\left(\Delta^{\otimes 2}(x)\right), \quad x \in U_{q_{i}}\left(s l_{2}\right),
$$

where $\Delta^{\prime}=\mathcal{P} \Delta \mathcal{P}$ and where $R$ is the junction $R$ matrix. From the relations above it is straightforward to show that:

$$
\mathrm{f}_{i}^{\otimes(N+1)}\left(\Delta^{\prime} \otimes(N+1)(x)\right) T(\lambda)=T(\lambda) \Delta^{\otimes(N+1)}\left(\mathrm{f}_{i}^{\otimes(N+1)}(x)\right),
$$

which leads, for trivial boundary conditions to

$$
\begin{gathered}
\mathrm{f}_{i}^{\otimes(N+1)}\left(\Delta^{\prime} \otimes(N+1)(x)\right) \mathbb{T}(\lambda)=\mathbb{T}(\lambda) \mathrm{f}_{i}^{\otimes(N+1)}\left(\Delta^{\prime \otimes(N+1)}(x)\right) \\
\Rightarrow \quad\left[t(\lambda), \mathrm{f}_{i}^{\otimes(N)}\left(\Delta^{\otimes(N)}(x)\right)\right]=0 .
\end{gathered}
$$

Hence, the transfer matrix with trivial boundary conditions enjoys the $\otimes_{i=0}^{3} U_{q_{i}}\left(s l_{2}\right)$ symmetry (similarly it may be shown that the transfer matrix enjoys the obvious symmetry $\otimes_{i=1}^{3} U_{a_{i}}\left(s l_{2}\right)$, see also $[8,6]$ for further details). The statement may be generalized for any $\mathrm{f}_{i_{1} \ldots i_{m}}$ representation $\forall n$ provided that relations (10) are valid:

$$
\left[t(\lambda), \mathrm{f}_{i_{1} \ldots i_{m}}^{\otimes(N)}\left(\Delta^{\otimes(N)}(x)\right)\right]=0, \quad x \in U_{q_{i_{1} \ldots i_{m}}}\left(s l_{2}\right) .
$$

We shall focus here, since it is more interesting, on the breaking of the non trivial symmetries expressed via the representations $\mathrm{f}_{i}$. The existence of a non-trivial "boundary" may couple the various $V_{l^{(i)}}$ spaces. In this case various choices of representations of the boundary TemperleyLieb algebra lead to consistent breaking of the original symmetry in different ways. Particularly, focus first on the $n=3$ case and define

$$
K^{(i)}=x(\lambda)+y(\lambda) M_{i} \quad \text { and } \quad \mathbb{T}^{(i)}=T(\lambda) K^{(i)}(\lambda) T^{-1}(-\lambda) .
$$

It is clear bearing also in mind the results of Section 3 that

$$
\begin{aligned}
& {\left[K^{(i)}(\lambda), \mathrm{f}_{i}\left(\mathcal{Q}_{i}\right) \otimes_{j \neq i} \mathrm{f}_{j}(x)\right]=0} \\
& \quad \Rightarrow \mathbb{T}^{(i)}(\lambda) \mathrm{f}_{i}^{\otimes(N+1)}\left(\Delta^{\prime}(N+1)\left(\mathcal{Q}_{i}\right)\right) \otimes_{j \neq i} \mathrm{f}_{j}^{\otimes(N+1)}\left(\Delta^{\prime}(N+1)(x)\right) \\
& \quad=\mathrm{f}_{i}^{\otimes(N+1)}\left(\Delta^{\prime(N+1)}\left(\mathcal{Q}_{i}\right)\right) \otimes_{j \neq i} \mathrm{f}_{j}^{\otimes(N+1)}\left(\Delta^{\prime(N+1)}(x)\right) \mathbb{T}^{(i)}(\lambda),
\end{aligned}
$$

and it is then shown that

$$
\left[t^{(i)}(\lambda), \mathrm{f}_{i}^{\otimes N}\left(\Delta^{(N)}\left(\mathcal{Q}_{i}\right)\right) \otimes_{j \neq i} \mathrm{f}_{j}^{\otimes N}\left(\Delta^{(N)}(x)\right)\right]=0, \quad x \in U_{q_{j}}\left(s l_{2}\right) .
$$

With this statement we basically conclude our study on the symmetries of the junction spin chain with trivial and non-trivial integrable boundary conditions. Again the boundary symmetry statement can be generalized for any representation $\mathrm{f}_{i_{1} \ldots i_{m}}, \forall n$ provided that relations (10), (16) hold. Indeed, set

$$
K^{\left(i_{1} \ldots i_{m}\right)}=x(\lambda)+y(\lambda) M_{i_{1} \ldots i_{m}}, \quad t^{\left(i_{1} \ldots i_{m}\right)}(\lambda)=\operatorname{tr}\left\{M T(\lambda) K^{\left(i_{1} \ldots i_{m}\right)}(\lambda) T^{-1}(-\lambda)\right\} .
$$


Then it is straightforward to show, provided that (10), (16) are valid:

$$
\begin{aligned}
& {\left[t^{\left(i_{1} \ldots i_{m}\right)}(\lambda), \mathrm{f}_{i_{1} \ldots i_{m}}^{\otimes N}\left(\Delta^{(N)}\left(\mathcal{Q}_{i_{1} \ldots i_{m}}\right)\right) \otimes_{\left\{j_{1}, \ldots, j_{m}\right\} \neq\left\{i_{1}, \ldots i_{m}\right\}} \mathrm{f}_{j_{1} \ldots j_{m}}^{\otimes N}\left(\Delta^{(N)}(x)\right)\right]=0,} \\
& \quad x \in U_{q_{j_{1} \ldots j_{m}}}\left(s l_{2}\right) .
\end{aligned}
$$

Since the junction representation is a representation of the (boundary) TL algebra as is the XXZ one expects, according to the general discussion in [7], that the spectra of the models will by identical, up to multiplicities, (see universal expressions for Hamiltonians in [7] and generic expressions of open transfer matrices in terms of elements of the (boundary) Temperley-Lieb algebra in [6]). Note that the results of the present section are explicitly proven provided that the results, and conjectures of Sections 2.1, 2.2, 3 and 3.1 are valid.

\section{Discussion}

We have been able to identify a novel family of representations of the Temperley-Lieb algebra, the so called junction representations, which involve $\mathrm{n}$ copies of the familiar XXZ representation. Interestingly enough, it turns out that the number of exact symmetries associated to the junction representation is drastically increased with the number $n$. The existence of the extended junction representation in the case of the boundary Temperley-Lieb algebra is examined, and particular non-trivial "boundary" elements are identified. The results on the boundary case are non-trivial and analytic proof is provided in the case where $n=3$, whereas in the general case a conjecture on the form of the representation of the boundary element is stated, and is checked with Mathematica for $n=4,5$. The associated residual symmetries in this case are also extracted.

More precisely, regarding the study of the associated symmetries: we explicitly show in Section 2.1 that in addition to the expected symmetries particular non-trivial algebra realizations commute with the junction representation, whereas in Section 3 we state a generic conjecture on the existence of a big number of non-trivial symmetry algebras, which we support qualitatively. We check however the validity of our conjecture with Mathematica up to $n=5$. Similar results obtained in Section 3.1 concerning the associated boundary symmetries.

What is highly non-trivial in the present investigation in the existence of a large number of non-trivial exact symmetry algebras. It is important to note that for the moment there is no-rigorous proof that these consist the full symmetry algebra associated to the junction representation. Nevertheless, it would be very interesting if one could find an extra symmetry that does not fall to the categories discussed in the present study. Moreover, the obvious number of associated symmetries does not seem to coincide with the number of the obtained non-trivial symmetries, and this is also an intriguing issue, which is still open even in the simplest case $n=2$, the "asymmetric twin" representation [7]. We hope to address these significant issues in future investigations.

Using this particular representation we constructed the corresponding novel physical quantum system, that is the "junction" quantum spin chain, and examined the associated exact symmetries. Within this spirit an interesting problem to pursue is the study of the aforementioned representations, and symmetries in the context of other statistical physics, such as face or loop models. An even more intriguing question is the possible existence of some kind of continuum limit of the junction spin chain, which also enjoys the big number of exact symmetries identified here. More precisely, universal local Hamiltonians in terms of the elements of the (boundary) Temperley-Lieb algebras are available (see e.g. [7, 8]), thus one may try to obtain the so called long wave length continuum limit (see e.g. [9]) of such expressions, that is obtain classical continuum Hamiltonians. We shall fully address these and related issues in forthcoming publications. 


\section{A Parametrization and solution of the Hecke condition}

In this appendix we present how one obtains solutions of the quadratic constraint of the Temperley-Lieb algebra (Hecke condition)

$$
\mathbb{U}^{2}=-\left(q+q^{-1}\right) \mathbb{U}
$$

We focus first for simplicity on the case $n=3$. A detailed study for the generic solution will be presented elsewhere.

By taking into account the form of the junction representation, the Hecke condition is written as

$$
\begin{array}{r}
a_{1} a_{2} a_{3}+a_{1}^{-1} a_{2}^{-1} a_{3}^{-1}+a_{1} a_{2} a_{3}^{-1}+a_{1} a_{2}^{-1} a_{3}+a_{1}^{-1} a_{2} a_{3} \\
+a_{1}^{-1} a_{2}^{-1} a_{3}+a_{1}^{-1} a_{2} a_{3}^{-1}+a_{1} a_{2}^{-1} a_{3}^{-1}=q+q^{-1} .
\end{array}
$$

It is now convenient to parametrize $a_{i}$ as follows:

$$
a_{i}=z_{i} q^{\frac{1}{3}}, \quad i \in\{1,2,3\}
$$

In addition, if we set

$$
a_{1} a_{2} a_{3}=q \quad \Rightarrow \quad a_{1}^{-1} a_{2}^{-1} a_{3}^{-1}=q^{-1},
$$

then the rest terms sum up to give zero. Hence we are left with three equations to solve in order to identify the three unknown quantities $z_{i}$, which read

$$
\begin{aligned}
& z_{1} z_{2} z_{3}=1, \quad z_{1} z_{2} z_{3}^{-1}+z_{1} z_{2}^{-1} z_{3}+z_{1}^{-1} z_{2} z_{3}=0 \\
& z_{1}^{-1} z_{2}^{-1} z_{3}+z_{1}^{-1} z_{2} z_{3}^{-1}+z_{1} z_{2}^{-1} z_{3}^{-1}=0 .
\end{aligned}
$$

Solving the equations above one can explicitly fix the values of $z_{i}$, for the particular parametrization of $a_{i}$. Of course one may choose a different parametrization, but in any case these should be equivalent. A simple solution of the above system of equations is the following:

$$
z_{1}=e^{\frac{i \pi}{3}}, \quad z_{2}=e^{\frac{2 i \pi}{3}}, \quad z_{3}=e^{i \pi} .
$$

It is straightforward to check that the above solution satisfies the system (17).

Let us now generalize our comments for every $n>3$. We start from the generic polynomial condition

$$
(-1)^{n} \prod_{j=1}^{n}\left(a_{j}+a_{j}^{-1}\right)=-\left(q+q^{-1}\right),
$$

and expanding the expressions above we take

$$
a_{1} a_{2} \cdots a_{n}+\sum_{i} a_{1} \cdots a_{i}^{-1} \cdots a_{n}+\sum_{i_{1}, i_{2}} a_{1} \cdots a_{i_{1}}^{-1} \cdots a_{i_{2}}^{-1} \cdots a_{n}+\cdots=(-1)^{n-1}\left(q+q^{-1}\right) .
$$

We parameterize, as in $n=3$ case, by setting

$$
q=(-1)^{n-1} a_{1} a_{2} \cdots a_{n}, \quad a_{j}=z_{j} q^{\frac{1}{n}} .
$$

Our task now is to identify the constants $z_{i}$. By also requiring, in addition to (18), the factor in front of every power $q^{\frac{m}{n}}(-n<m<n)$ to disappear we end up with a set of $n$ equations. More specifically, we end up with a system of $n$ unknown quantities, the $z_{j}$ 's and $n$ equations, so that in principle the system can be solved and $z_{j}$ 's can be determined. 


\section{B Proof for $n=3$}

As it has been already stated, we have checked the validity of our conjecture for the cases $n=4,5$ by using Mathematica. However, we were able to perform analytic calculations for the $n=3$ case. In this appendix, we describe part of our analytic computation.

It is convenient for what follows to write down the explicit expression of the each one of the $\mathrm{XXZ}$ representations for $n=3$ : i.e.

$$
\begin{aligned}
\rho_{a_{1}}\left(\mathbb{U}_{l^{(1)}}\right)= & \cdots \mathbb{I} \otimes\left(\sum_{a \neq b} e_{a b} \otimes \mathbb{I} \otimes \mathbb{I} \otimes e_{b a} \otimes \mathbb{I} \otimes \mathbb{I}\right. \\
& \left.-\sum_{a \neq b} a_{1}^{-\operatorname{sgn}(a-b)} e_{a a} \otimes \mathbb{I} \otimes \mathbb{I} \otimes e_{b b} \otimes \mathbb{I} \otimes \mathbb{I}\right) \otimes \mathbb{I} \cdots, \\
\rho_{a_{2}}\left(\mathbb{U}_{l^{(2)}}\right)= & \cdots \mathbb{I} \otimes\left(\sum_{a \neq b} \mathbb{I} \otimes e_{a b} \otimes \mathbb{I} \otimes \mathbb{I} \otimes e_{b a} \otimes \mathbb{I}\right. \\
& \left.-\sum_{a \neq b} a_{2}^{-\operatorname{sgn}(a-b)} \mathbb{I} \otimes e_{a a} \otimes \mathbb{I} \otimes \mathbb{I} \otimes e_{b b} \otimes \mathbb{I}\right) \otimes \mathbb{I} \cdots, \\
\rho_{a_{3}}\left(\mathbb{U}_{l^{(3)}}\right)= & \cdots \mathbb{I} \otimes\left(\sum_{a \neq b} \mathbb{I} \otimes \mathbb{I} \otimes e_{a b} \otimes \mathbb{I} \otimes \mathbb{I} \otimes e_{b a}\right. \\
& \left.-\sum_{a \neq b} a_{3}^{-\operatorname{sgn}(a-b)} \mathbb{I} \otimes \mathbb{I} \otimes e_{a a} \otimes \mathbb{I} \otimes \mathbb{I} \otimes e_{b b}\right) \otimes \mathbb{I} \cdots .
\end{aligned}
$$

Our purpose is to show that

$$
\left[\Theta\left(\mathbb{U}_{l}\right), f_{i}^{\otimes N}(\Delta(x))\right]=0, \quad x \in U_{q_{i}}\left(s l_{2}\right),
$$

for $i=0, \ldots, 3$. Let us focus for simplicity, and for saving writing on $N=2$, since it is then easy to generalize for any $N$ (see also [7]). We shall show in detail the computation for the $f_{0}$ representation, the rest follow in the same spirit. Consider first the element $e$ of the $U_{q_{0}}\left(s l_{2}\right)$ quantum algebra. The left-hand side of $\left[\Theta\left(\mathbb{U}_{1}\right), f_{0}^{\otimes 2}(\Delta(e))\right]$ is written explicitly as

$$
\begin{aligned}
& \Theta\left(\mathbb{U}_{1}\right) \mathrm{f}_{0}^{\otimes 2}(\Delta(e))=\left(\sum_{a \neq b} e_{a b} \otimes \mathbb{I} \otimes \mathbb{I} \otimes e_{b a} \otimes \mathbb{I} \otimes \mathbb{I}-\sum_{a \neq b} a_{1}^{-\operatorname{sgn}(a-b)} e_{a a} \otimes \mathbb{I} \otimes \mathbb{I} \otimes e_{b b} \otimes \mathbb{I} \otimes \mathbb{I}\right) \\
& \times\left(\sum_{m \neq n} \mathbb{I} \otimes e_{m n} \otimes \mathbb{I} \otimes \mathbb{I} \otimes e_{n m} \otimes \mathbb{I}-\sum_{m \neq n} a_{2}^{-\operatorname{sgn}(m-n)} \mathbb{I} \otimes e_{m m} \otimes \mathbb{I} \otimes \mathbb{I} \otimes e_{n n} \otimes \mathbb{I}\right) \\
& \quad \times\left(\sum_{k \neq l} \mathbb{I} \otimes \mathbb{I} \otimes e_{k l} \otimes \mathbb{I} \otimes \mathbb{I} \otimes e_{l k}-\sum_{k \neq l} a_{3}^{-\operatorname{sgn}(k-l)} \mathbb{I} \otimes \mathbb{I} \otimes e_{k k} \otimes \mathbb{I} \otimes \mathbb{I} \otimes e_{l l}\right) \\
& \times\left(\left(q_{0}^{-\frac{e_{11}}{2}} \otimes e_{11} \otimes e_{11}+q_{0}^{\frac{e_{22}}{2}} \otimes e_{22} \otimes e_{22}+\mathbb{I} \otimes e_{11} \otimes_{22}+\mathbb{I} \otimes e_{22} \otimes e_{11}\right)\right. \\
& \otimes e_{12} \otimes e_{12} \otimes e_{12}+\otimes e_{12} \otimes e_{12} \otimes e_{12} \\
& \left.\otimes\left(q_{0}^{\frac{e_{11}}{2}} \otimes e_{11} \otimes e_{11}+q_{0}^{-\frac{e_{22}}{2}} \otimes e_{22} \otimes e_{22}+\mathbb{I} \otimes e_{11} \otimes_{22}+\mathbb{I} \otimes e_{22} \otimes e_{11}\right)\right) .
\end{aligned}
$$

Using the property $e_{i j} e_{k l}=\delta_{j k} e_{i l}$ and after some quite cumbersome algebra we end up with the following expression for the first

$$
\Theta\left(\mathbb{U}_{1}\right) \mathrm{f}_{0}^{\otimes 2}(\Delta(e))=q_{0}^{\frac{1}{2}} e_{12} \otimes e_{12} \otimes e_{12} \otimes e_{22} \otimes e_{22} \otimes e_{22}
$$




$$
\begin{aligned}
& +q_{0}^{-\frac{1}{2}} e_{22} \otimes e_{22} \otimes e_{22} \otimes e_{12} \otimes e_{12} \otimes e_{12}-a_{3}^{-1} q_{0}^{\frac{1}{2}} e_{12} \otimes e_{12} \otimes e_{22} \otimes e_{22} \otimes e_{22} \otimes e_{12} \\
& -a_{3} q_{0}^{-\frac{1}{2}} e_{22} \otimes e_{2} \otimes e_{12} \otimes e_{12} \otimes e_{12} \otimes e_{22}-a_{2}^{-1} q_{0}^{\frac{1}{2}} e_{12} \otimes e_{22} \otimes e_{12} \otimes e_{22} \otimes e_{12} \otimes e_{22} \\
& -a_{2} q_{0}^{-\frac{1}{2}} e_{22} \otimes e_{12} \otimes e_{22} \otimes e_{12} \otimes e_{22} \otimes e_{12}+a_{2}^{-1} a_{3}^{-1} q_{0}^{\frac{1}{2}} e_{12} \otimes e_{22} \otimes e_{22} \otimes e_{22} \otimes e_{12} \otimes e_{12} \\
& +a_{2} a_{3} q_{0}^{-\frac{1}{2}} e_{22} \otimes e_{12} \otimes e_{12} \otimes e_{12} \otimes e_{22} \otimes e_{22}-a_{1}^{-1} q_{0}^{\frac{1}{2}} e_{22} \otimes e_{12} \otimes e_{12} \otimes e_{12} \otimes e_{22} \otimes e_{22} \\
& -a_{1} q_{0}^{-\frac{1}{2}} e_{12} \otimes e_{22} \otimes e_{22} \otimes e_{22} \otimes e_{12} \otimes e_{12}+a_{1}^{-1} a_{3}^{-1} q_{0}^{\frac{1}{2}} e_{22} \otimes e_{12} \otimes e_{22} \otimes e_{12} \otimes e_{22} \otimes e_{12} \\
& +a_{1} a_{2} q_{0}^{-\frac{1}{2}} e_{12} \otimes e_{22} \otimes e_{12} \otimes e_{22} \otimes e_{12} \otimes e_{12} \\
& +a_{1}^{-1} a_{2}^{-1} q_{0}^{\frac{1}{2}} e_{22} \otimes e_{22} \otimes e_{12} \otimes e_{12} \otimes e_{12} \otimes e_{22} \\
& +a_{1} a_{2} q_{0}^{-\frac{1}{2}} e_{12} \otimes e_{12} \otimes e_{22} \otimes e_{22} \otimes e_{22} \otimes e_{12} \\
& -a_{1}^{-1} a_{2}^{-1} a_{3}^{-1} q_{0}^{\frac{1}{2}} e_{22} \otimes e_{22} \otimes e_{22} \otimes e_{12} \otimes e_{12} \otimes e_{12} \\
& -a_{1} a_{2} a_{3} q_{0}^{-\frac{1}{2}} e_{12} \otimes e_{12} \otimes e_{12} \otimes e_{22} \otimes e_{22} \otimes e_{22} .
\end{aligned}
$$

and the second term of the commutator

$$
\begin{aligned}
\mathrm{f}_{0}^{\otimes 2}( & \Delta(e)) \Theta\left(\mathbb{U}_{1}\right)=q_{0}^{-\frac{1}{2}} e_{12} \otimes e_{12} \otimes e_{12} \otimes e_{11} \otimes e_{11} \otimes e_{11} \\
& +q_{0}^{\frac{1}{2}} e_{11} \otimes e_{11} \otimes e_{11} \otimes e_{12} \otimes e_{12} \otimes e_{12}-a_{3} q_{0}^{-\frac{1}{2}} e_{12} \otimes e_{12} \otimes e_{11} \otimes e_{11} \otimes e_{11} \otimes e_{12} \\
& -a_{3}^{-1} q_{0}^{\frac{1}{2}} e_{11} \otimes e_{11} \otimes e_{12} \otimes e_{12} \otimes e_{12} \otimes e_{11}-a_{2} q_{0}^{-\frac{1}{2}} e_{12} \otimes e_{11} \otimes e_{12} \otimes e_{11} \otimes e_{12} \otimes e_{11} \\
& -a_{2}^{-1} q_{0}^{\frac{1}{2}} e_{11} \otimes e_{12} \otimes e_{11} \otimes e_{12} \otimes e_{11} \otimes e_{12}+a_{2} a_{3} q_{0}^{-\frac{1}{2}} e_{12} \otimes e_{11} \otimes e_{11} \otimes e_{11} \otimes e_{12} \otimes e_{12} \\
& +a_{2}^{-1} a_{3}^{-1} q_{0}^{\frac{1}{2}} e_{11} \otimes e_{12} \otimes e_{12} \otimes e_{12} \otimes e_{11} \otimes e_{11}-a_{1} q_{0}^{-\frac{1}{2}} e_{11} \otimes e_{12} \otimes e_{12} \otimes e_{12} \otimes e_{11} \otimes e_{11} \\
& -a_{1}^{-1} q_{0}^{\frac{1}{2}} e_{12} \otimes e_{11} \otimes e_{11} \otimes e_{11} \otimes e_{12} \otimes e_{12}+a_{1} a_{3} q_{0}^{-\frac{1}{2}} e_{11} \otimes e_{12} \otimes e_{11} \otimes e_{12} \otimes e_{11} \otimes e_{12} \\
& +a_{1}^{-1} a_{3}^{1} q_{0}^{\frac{1}{2}} e_{12} \otimes e_{11} \otimes e_{12} \otimes e_{11} \otimes e_{12} \otimes e_{11}+a_{1} a_{2} q_{0}^{-\frac{1}{2}} e_{11} \otimes e_{11} \otimes e_{12} \otimes e_{12} \otimes e_{12} \otimes e_{11} \\
& +a_{1}^{-1} a_{2}^{-1} q_{0}^{\frac{1}{2}} e_{12} \otimes e_{12} \otimes e_{11} \otimes e_{11} \otimes e_{11} \otimes e_{12} \\
& -a_{1} a_{2} a_{2} q_{0}^{-\frac{1}{2}} e_{11} \otimes e_{11} \otimes e_{11} \otimes e_{12} \otimes e_{12} \otimes e_{12} \\
& -a_{1}^{-1} a_{2}^{-1} a_{3}^{-1} q_{0}^{\frac{1}{2}} e_{12} \otimes e_{12} \otimes e_{12} \otimes e_{11} \otimes e_{11} \otimes e_{11} .
\end{aligned}
$$

Now notice that every two terms in each side of the commutator are canceled provided that $q_{0}=a_{1} a_{2} a_{3}$. This result has also been found to hold for the $n=4,5$ cases; each side of the commutator vanishes identically, for the appropriate form of $q_{i}, q_{i_{1} i_{2}}$.

The commutator of the junction representation with $\mathrm{f}_{0}^{\otimes 2}(\Delta(f))$ follows in the same spirit and the expressions obtained are very similar to those presented in (19) and (20). However, the commutator of the representation with $\mathrm{f}_{0}^{\otimes 2}(\Delta(h))$ is even easier to cope with, since it gives identically zero, without requiring any particular form for the $q_{i}$. This has also been outlined in the general comments of Section 2.2, where the relation $\left[\Theta\left(\mathbb{U}_{1}\right), \mathrm{f}_{i_{1} \ldots i_{m}}^{\otimes N}\left(\Delta^{(N)}(h)\right)\right]=0$ has been proved there by using some simple heuristic arguments. In the same spirit the proof can be extended for $i=1,2,3(n=3)$.

\section{Explicit expressions for $n=4$}

We present in this Appendix explicit expressions of the representations for the $n=4$ case to further illustrate the structure of these representations. We also write down the explicit value 
of $q_{i}, q_{i j}$ so that the respective representation is a quantum symmetry of our algebra

$$
\begin{aligned}
& \mathrm{f}_{0}(e)=e_{12} \otimes e_{12} \otimes e_{12} \otimes e_{12}, \quad \mathrm{f}_{0}(f)=e_{21} \otimes e_{21} \otimes e_{21} \otimes e_{21}, \\
& \mathrm{f}_{0}(h)=e_{11} \otimes e_{11} \otimes e_{11} \otimes e_{11}-e_{22} \otimes e_{22} \otimes e_{22} \otimes e_{22}, \quad q_{0}=-a_{1} a_{2} a_{3} a_{4}, \\
& \mathrm{f}_{1}(e)=e_{21} \otimes e_{12} \otimes e_{12} \otimes e_{12}, \quad \mathrm{f}_{1}(f)=e_{12} \otimes e_{21} \otimes e_{21} \otimes e_{21}, \\
& \mathrm{f}_{1}(h)=e_{22} \otimes e_{11} \otimes e_{11} \otimes e_{11}-e_{11} \otimes e_{22} \otimes e_{22} \otimes e_{22}, \quad q_{1}=-a_{1}^{-1} a_{2} a_{3} a_{4}, \\
& \mathrm{f}_{2}(e)=e_{12} \otimes e_{21} \otimes e_{12} \otimes e_{12}, \quad \mathrm{f}_{2}(f)=e_{21} \otimes e_{12} \otimes e_{21} \otimes e_{21}, \\
& \mathrm{f}_{2}(h)=e_{11} \otimes e_{22} \otimes e_{11} \otimes e_{11}-e_{22} \otimes e_{11} \otimes e_{22} \otimes e_{22}, \quad q_{2}=-a_{1} a_{2}^{-1} a_{3} a_{4}, \\
& \mathrm{f}_{3}(e)=e_{12} \otimes e_{12} \otimes e_{21} \otimes e_{12}, \quad \mathrm{f}_{3}(f)=e_{21} \otimes e_{21} \otimes e_{12} \otimes e_{21}, \\
& \mathrm{f}_{3}(h)=e_{11} \otimes e_{11} \otimes e_{22} \otimes e_{11}-e_{22} \otimes e_{22} \otimes e_{11} \otimes e_{22}, \quad q_{3}=-a_{1} a_{2} a_{3}^{-1} a_{4}, \\
& \mathrm{f}_{4}(e)=e_{12} \otimes e_{12} \otimes e_{12} \otimes e_{21}, \quad \mathrm{f}_{4}(f)=e_{21} \otimes e_{21} \otimes e_{21} \otimes e_{12}, \\
& \mathrm{f}_{4}(h)=e_{11} \otimes e_{11} \otimes e_{11} \otimes e_{22}-e_{22} \otimes e_{22} \otimes e_{22} \otimes e_{11}, \quad q_{4}=-a_{1} a_{2} a_{3} a_{4}^{-1}, \\
& \mathrm{f}_{34}(e)=e_{12} \otimes e_{12} \otimes e_{21} \otimes e_{21}, \quad \mathrm{f}_{34}(f)=e_{21} \otimes e_{21} \otimes e_{12} \otimes e_{12}, \\
& \mathrm{f}_{34}(h)=e_{11} \otimes e_{11} \otimes e_{22} \otimes e_{22}-e_{22} \otimes e_{22} \otimes e_{11} \otimes e_{11}, \quad q_{34}=-a_{1} a_{2} a_{3}^{-1} a_{4}^{-1}, \\
& \mathrm{f}_{24}(e)=e_{12} \otimes e_{21} \otimes e_{12} \otimes e_{21}, \quad \mathrm{f}_{24}(f)=e_{21} \otimes e_{12} \otimes e_{21} \otimes e_{12}, \\
& \mathrm{f}_{24}(h)=e_{11} \otimes e_{22} \otimes e_{11} \otimes e_{22}-e_{22} \otimes e_{11} \otimes e_{22} \otimes e_{11} \quad q_{24}=-a_{1} a_{2}^{-1} a_{3} a_{4}^{-1}, \\
& \mathrm{f}_{23}(e)=e_{12} \otimes e_{21} \otimes e_{21} \otimes e_{12}, \quad \mathrm{f}_{23}(f)=e_{21} \otimes e_{12} \otimes e_{12} \otimes e_{21}, \\
& \mathrm{f}_{23}(h)=e_{11} \otimes e_{22} \otimes e_{22} \otimes e_{11}-e_{22} \otimes e_{11} \otimes e_{11} \otimes e_{22}, \quad q_{23}=-a_{1} a_{2}^{-1} a_{3}^{-1} a_{4} .
\end{aligned}
$$

\section{Acknowledgements}

NK acknowledges financial support provided by the Research Committee of the University of Patras via a K.Karatheodori fellowship under contract number C.915, and partial support by the LLP/Erasmus Placements 2009-2010 program with contract 0099/2009. He would also like to thank the CPhT of Ecole Polytechnique for kind hospitality and partial support by the ERC Advanced Grant 226371, the ITN programme PITN- GA-2009-237920 and the IFCPAR CEFIPRA programme 4104-2 during the completion of this work.

\section{References}

[1] Baxter R.J., Exactly solved models in statistical mechanics, Academic Press, London, 1982.

Baxter R.J., Partition function of the eight vertex lattice model, Ann. Physics 70 (1972), 193-228.

Baxter R.J., Asymptotically degenerate maximum eigenvalues of the eight-vertex model transfer matrix and interfacial tension, J. Statist. Phys. 8 (1973), 25-55.

[2] Bourbaki N., Groupes et algèbres de Lie, Ch. 4, Exerc. 22-24, Hermann, Paris, 1968.

[3] Cherednik I., A unification of Knizhnik-Zamolodchikov and Dunkl operators via affine Hecke algebras, Invent. Math. 106 (1991), 411-431.

[4] Cherednik I.V., Factorizing particles on a half line and root systems, Theoret. and Math. Phys. 61 (1984), 977-983.

[5] Delius G., Mackay N., Quantum group symmetry in sine-Gordon and affine Toda field theories on the half-line, Comm. Math. Phys. 233 (2003), 173-190, hep-th/0112023.

[6] Doikou A., Boundary non-local charges from the open spin chain, J. Stat. Mech. Theory Exp. 2005 (2005), P12005, 17 pages, math-ph/0402067.

Doikou A., Asymmetric twin representation: the transfer matrix symmetry, SIGMA 3 (2007), 009, 19 pages, math-ph/0606040.

[7] Doikou A., Martin P.P., Hecke algebraic approach to the reflection equation for spin chains, J. Phys. A: Math. Gen. 36 (2003), 2203-2225, hep-th/0206076.

Doikou A., Martin P.P, On quantum group symmetry and Bethe ansatz for the asymmetric twin spin chain with integrable boundary, J. Stat. Mech. Theory Exp. 2006 (2006), P06004, 43 pages, hep-th/0503019. 
[8] Doikou A., From affine Hecke algebras to boundary symmetries, Nuclear Phys. B 725 (2005), 493-530, math-ph/0409060.

[9] Fradkin E.H., Field theories of condensed matter systems, Frontiers in Physics, Vol. 82, Addison-Wesley, Redwood City, CA, 1991.

[10] Graham J.J., Lehrer G.I., Cellular algebras, Invent. Math. 123 (1996), 1-34.

[11] Isaev A.P., Ogievetsky O.V., On Baxterized solutions of reflection equation and integrable chain models, Nuclear Phys. B $\mathbf{7 6 0}$ (2007), 167-183, math-ph/0510078.

Isaev A.P., Ogievetsky O.V., Os'kin A.F., Chain models on Hecke algebra for corner type representations, Rep. Math. Phys. 61 (2008), 311-317, arXiv:0710.0261.

[12] Jimbo M., Quantum $R$ matrix for the generalized Toda system, Comm. Math. Phys. 102 (1986), 537-547.

[13] Jimbo M., A q-difference analogue of $U(g)$ and the Yang-Baxter equation, Lett. Math. Phys. 10 (1985), 63-69.

Jimbo M., A q-analogue of $U(g l(N+1))$, Hecke algebra, and the Yang-Baxter equation, Lett. Math. Phys. 11 (1986), 247-252.

[14] Kazhdan D., Lusztig G., Representations of Coxeter groups and Hecke algebras, Invent. Math. 53 (1979), $165-184$.

[15] Kulish P.P., Mudrov A.I., Baxterization of solutions to reflection equation with Hecke R-matrix, Lett. Math. Phys. 75 (2006), 151-170, math.QA/0508289.

Kulish P.P., Manojlovic N., Nagy Z., Quantum symmetry algebras of spin systems related to Temperley-Lieb R-matrices, J. Math. Phys. 49 (2008), 023510, 9 pages, arXiv:0712.3154.

[16] Levy D., Martin P.P., Hecke algebra solutions to the reflection equation, J. Phys. A: Math. Gen. 27 (1994), L521-L526,

Martin P.P., Woodcock D., Levy D., A diagrammatic approach to Hecke algebras of the reflection equation, J. Phys. A: Math. Gen. 33 (2000), 1265-1296.

[17] Martin P.P., Woodcock D., Generalized blob algebras and alcove geometry, LMS J. Comput. Math. 6 (2003), 249-296, math.RT/0205263.

Martin P.P., Saleur H., The blob algebra and the periodic Temperley-Lieb algebra, Lett. Math. Phys. 30 (1994), 189-206, hep-th/9302094.

[18] Martin P.P., Potts models and related problems in statistical mechanics, Series on Advances in Statistical Mechanics, Vol. 5, World Scientific Publishing Co., Inc., Teaneck, NJ, 1991.

[19] Mezincescu L., Nepomechie R.I., Fractional-spin integrals of motion for the boundary sine-Gordon model at the free fermion point, Internat. J. Modern Phys. A 13 (1998), 2747-2764, hep-th/9709078.

[20] Nichols A., Spectral equivalences and symmetry breaking in integrable $S U_{q}(N)$ spin chains with boundaries, J. Stat. Mech. Theory Exp. 2005 (2005), P09009, 31 pages, hep-th/0507209.

de Gier J., Nichols A., The two-boundary Temperley-Lieb algebra, J. Algebra 321 (2009), 1132-1167, math.RT/0703338.

[21] Ram A., Ramagge J., Affine Hecke algebras, cyclotomic Hecke algebras and Clifford theory, in A Tribute to C.S. Seshadri (Chennai, 2002), Trends Math., Birkhäuser, Basel, 2003, 428-466, math.RT/0401322.

Ram A., Affine Hecke algebras and generalized standard Young tableaux, J. Algebra 260 (2003), 367-415, math.RT/0401329.

[22] Read N., Saleur H., Associative-algebraic approach to logarithmic conformal field theories, Nuclear Phys. B 777 (2007), 316-351, hep-th/0701117.

Pearce P.A., Rasmussen J., Ruelle P., Integrable boundary conditions and $W$-extended fusion in the logarithmic minimal models $L M(1, p)$, J. Phys. A: Math. Theor. 41 (2008), 295201, 16 pages, arXiv:0803.0785.

[23] Sklyanin E.K., Boundary conditions for integrable quantum systems, J. Phys. A: Math. Gen. 21 (1988), $2375-2389$.

[24] Temperley H.N.V., Lieb E.H., Relations between the 'percolation' and 'colouring' problem and other graphtheoretical problems associated with regular planar lattices: some exact results for the 'percolation' problem, Proc. Roy. Soc. London Ser. A 322 (1971), 251-280. 